\title{
Psychotherapy - a survey of psychiatrists' attitudes, beliefs and practice
}

\author{
Sue Bradbury, L. S. O'Brien and Michael Göpfert
}

\begin{abstract}
A postal survey was conducted among doctors practising poychictiry in the Mersey Region to ascertain atthudes, bellets and proctice with regard to psychotherapy, achleving a response rate of over $60 \%$. There was a surprising cbsence of very strong feellings for or against poychotherapy which was not seen as a powertul treatment modallty. There was little knowledge of the facllities provided by the regional service, and no ceneral support for their expansion. The role of training in poychotherapy and its stonificance in light of guidelines for general trainees are discuseed. A need is recognised for greater awareness and contact between poychiatrists and the poychotherapy service.
\end{abstract}

Psychotherapy services in the Mersey Reglon have a relatively short history. Prior to the first consultant appointment in 1987, patients in need of psychotherapy either received it ad hoc within their local service, or were treated by some other means. In fact Merseyside psychiatrists had survived for many years without a specialist service and many still appear to have little contact. In 1990-91 approximately half of the referrals were from psychiatrists.

Initially the psychotherapy service in central Liverpool had both district and regional responsibilities. The role is now more that of a district based service. There is an intentionally eclectic approach, with emphasis on behavioural, systems, and dynamic therapies, both individual and group. The service is relatively small. At the time of the survey it was co-located with the Academic Department of Psychiatry and an acute in-patient unit, although it has now moved to a separate location.

A major demand on such a service is teaching psychotherapy. With the recent publication of the College guidelines for training of junior doctors (Grant et al, 1993). it is envisaged that in the future all psychiatrists will have a working knowledge of psychotherapy. One would hope that this would increase the trainees' therapeutic repertoire and the conviction that psychotherapy is a potent and useful treatment. In Liverpool there are part-time and full-time placements available for general and higher professional trainees. Other trainees may have direct contact for supervision.

The purpose of the study was threefold: to increase the profile of the service among colleagues; to identify liaison and educational needs; and to provide information for optimal strategic planning of the service for the future.

\section{The study}

\section{Subjects}

We surveyed all doctors in the region who were in a psychiatric post during February 1991 ( $n=237$ ). This included trainees, staff grades and vocational trainees. After two mailings a total of 149 completed questionnaires were received, giving a response rate of $62.8 \%$.

\section{Questionnaire}

After enquiring into demographic detail, the first section of the questionnaire dealt with psychiatrists' views of their own practice orientation, opinions about what psychotherapy is and views on the role and relative values of different aspects of psychotherapy training. The second section dealt with views on the relationship of psychotherapy services to other areas of psychiatric practice, what specific treatments should be offered and to whom. The third dealt with their own relationship with the existing service and what they believed to be available, and the last was on their own current practice of psychotherapy. Most questions were posed as statements with an accompanying 5-point analogue scale. Some questions were posed as pick lists. (Details available on request). Non-parametric statistical tests of frequency comparison were used throughout, chi-squared with Yates's correction and Fisher's exact two-talled test. 


\section{Results}

Eighty-three (55.7\%) respondents were male and 66 (44.3\%) were female. The distribution by grade and speciality are shown in Tables 1 and 2 respectively. The response rate for general practice trainees was low, and possibly reflects either a lack of investment in psychiatric matters or a belief that the questionnaire did not apply to them.

Overall, there was a non-significant tendency for women to be younger, of junior grade, and of less years experience. The typical respondent was a consultant in general psychiatry in his thirties and with more than ten but less than 20 years experience (32.4\%). There were no significant differences in responses according to grade. speciality, gender, age or experience. There was a non-significant trend for those who had been practising longer to say their work was psychotherapeutically orientated. In general, for the majority of questions there was an absence of strong feeling with a large number of neutral responses.

One of the central questions to the survey was whether or not psychotherapy was believed to be a powerful treatment modality. Just over 20\% thought that it was while an equal number believed that it was not; the majority of respondents were neutral. Only $14 \%$ thought that all patients needed some sort of psychotherapeutic input, while twice as many thought that all patients did not. Those believing that psychotherapy was a potent treatment modality were more likely to have training in psychotherapy (Fisher's exact test, $P<0.05$ ), state their own practice was psychotherapeutically

Table 1. Distribution of psychiatrists by grade

\begin{tabular}{lr}
\hline Grade & \multicolumn{1}{c}{$\%$} \\
\hline Consultant & 41.1 \\
Staff grade & 6.1 \\
Senlor registrar & 13.7 \\
Registrar & 24.0 \\
Senlor house officer (SHO) & 14.4 \\
GPVT (general practice SHO) & 0.7 \\
\hline
\end{tabular}

Table 2. Distribution by speciallity

\begin{tabular}{lc}
\hline Speciaility & \% \\
\hline General psychiatiy & 54.4 \\
Child and adolescent & 16.5 \\
Old age psychiatiy & 9.7 \\
Leaming disabillities & 9.0 \\
Substance misuse & 4.1 \\
Forensic & 4.1 \\
Psychotherapy & 2.5
\end{tabular}

orlentated (Fisher's exact test, $P<0.00001$ ), and believe all patients need some psychotherapeutic input (Flsher's exact test, $P<0.002$ ). Whether people felt treatment was potent or not was not affected by time in psychiatry.

Of the respondents $56.4 \%$ had had either a job with a significant recognised psychotherapy component or a postgraduate course (course $16.1 \%$, job $19.5 \%$, both $20.8 \%$ ). There was a tendency for those with experience to see psychotherapy as more potent but this did not reach significance except for those who had completed both a job and a course (Fisher's exact test, $P<0.05)$. Psychiatrists with training in psychotherapy were much more likely to consider their own practice to be psychotherapeutically oriented $(P<0.002)$.

The vast majority of respondents thought postgraduate training in psychotherapy should be part of the curriculum for psychiatrists. They were asked to rank order a number of different methods of gaining psychotherapy experience. Over $60 \%$ ranked individual supervision first, followed by group superviston. trainee attachments, review of recorded sessions, qualifying course, seminars, personal psychotherapy, and no formal training.

Respondents seemed to have more definite opinions about the functions and relationship of a psychotherapy service to other psychiatric services than about the value of psychotherapy. Forty-three per cent said that psychotherapy should not be a separate spectalist service while only $15 \%$ thought it should provide a treatment service in its own right (like physiotherapy). Psychiatric assessment prior to referral was endorsed by $61 \%$, and $53 \%$ said the psychotherapy service should not take full psychiatric responsibility for the patient. Eighty-nine per cent thought that the service should not provide all therapy, and $71 \%$ thought it should not provide all psychotherapy supervision. Overall, the consensus (in rank order) on functions of the service was treating patients, consultation, assessment and selection, supervision, teaching and research. Those who had been in psychiatry over ten years were reluctant to rank any function higher than another.

Respondents were not strongly in favour of the provision of any particular form of psychotherapeutic treatment, with no single treatment being endorsed by more than a third of respondents. For no treatments other than brief dynamic, long-term, and family therapies were more respondents for than against. More than $50 \%$ were against psychodrama and supporttve psychotherapy. The rank order of preference was group, brief psychodynamic, family, cognitive, marital, behavioural, long-term, psychodrama, and supportive. Those who considered psychotherapy a potent treatment modality were 
significantly more likely to endorse the provision of the psychodynamic therapies; brief dynamic (Fisher's exact test, $P<0.02)$, long-term (Fisher's exact test. $P<0.00001$ ) and group therapy (Fisher's exact test, $P<0.00001$ ).

Psychotherapy was thought most useful for treating neurosis (40.2\%), personality disorder (23.5\%), relationship problems (34.2\%), sexual problems $(27.5 \%)$, and psychosomatic problems (20.8\%) but thought not to be of value in substance misuse (45\%), bipolar affective disorder (63\%), psychosis $(74.5 \%)$, and dementia $(80.5 \%)$. Those who thought psychotherapy is potent were more likely than to endorse treatment for neurosis (Fisher's exact test, $P<0.036$ ), personality disorder $\quad(P<0.0005)$, relationship problems $(P<0.015)$, sexual problems $(P<0.01)$, substance misuse $(P<0.004)$, psychosomatic disorders $(P<0.0005)$, and depression $(P<0.00001)$. This group was also significantly more likely to endorse psychotherapy for a wider range of subjects: children $(P<0.0002)$, elderly people $(P<0.001)$, learning disabllities $(P<0.037)$, those with a high IQ $(P<0.014)$, high socioeconomic class $(P<0.034)$, and low socioeconomic class $(P<0.03)$.

In terms of service usage, $8 \%$ frequently referred while $86 \%$ said they did not. Most thought the service should not expand (53.7\%) and should not take greater responsibility for patients $(66.4 \%)$. There was a general indecision or neutrality about the value or desirability of more contact with psychiatric teams. Belief in the potency or otherwise of psychotherapy did not affect any of these answers.

Respondents were asked to identify which therapies were available from the psychotherapy service. Therapies correctly identified were brief dynamic (55.7\%), group psychotherapy (52.3\%), long-term, $(50 \%)$, family therapy $(50 \%)$, behavioural (30.9\%), and psychodrama (19.5\%); those not available were also identified: cognitive (38.2\%), marital (35.6\%), sexual therapy $(23.5 \%)$, psychoanalysis $(19.5 \%)$, and transactional analysis (12\%). A minority (14\%) thought some other therapies should be offered which were not. Previous training in psychotherapy was not associated with a particular response pattern.

When asked about their own practice $75.5 \%$ spent less than 5 hours practising psychotherapy per week, with $12.5 \%$ spending 5-10 hours and $12 \%$ over 10 hours. Of those in psychiatry for more than three years, $41 \%$ spent less time practising psychotherapy than three years ago. Of these $94 \%$ said it was due to time constraints. $30 \%$ to others providing the service, $16 \%$ to seeing fewer cases, $16 \%$ to lack of facilities, $10 \%$ to the increased efficacy of other treatments and $6 \%$ to failure of efficacy of psychotherapy.

We asked subjects to describe their own psychotherapeutic practice. Descriptions were not readily classifiable, but the most common words used were 'supportive' 31\%, 'brief 25\%, 'individual' 22\%, 'cognittve' 16\%, 'behavioural' $14 \%$, and 'dynamic' $12 \%$. Only $19.5 \%$ sald they practised a particular school or model: cognitivebehavioural (7), eclectic (6), Hosbon conversational (4), psychodynamic (3), psychoanalytic (2), family (2), systemic (2), Rogerian (2), and one each gave Freudian, biological, structural, Malan, non-directive, object relations, group analysis, Kleinian, Foulkesian, mottvational, and supportive.

Respondents provided some informattve comments. One commented that psychotherapy was too expensive to be available on the National Health Service. Two had found the questionnaire difficult due to confusion over what psychotherapy is. Others wanted to know more about the service, and one sent us a booklet on his own servicel

\section{Comment}

The findings of this survey may not be readily applicable to other populations, as these will vary according to prevailing psychotherapy culture. Nevertheless the results illustrate a number of points with relevance to service planning. The response rate was relatively high for a postal survey.

The most striking aspect of the responses was the lack of strong feelings, as reflected in the questionnaire responses. This may be due in part to the tendency to respond centrally on a five point scale but probably also demonstrates genuine ambivalence among psychiatrists about the meaning and significance of psychotherapy. Approximately half of respondents claimed some postgraduate experience of psychotherapy.

Psychiatrists will refer to a service only if they believe the treatment offered is effective, and only $21.5 \%$ thought it was. Belief in psychotherapy was significantly associated with support of the psychodynamic therapies. However, the study shows that there are other reasons for lack of referral, including lack of awareness of the existence of the service, some lack of knowledge about indications for treatment, and lack of knowledge of the services offered. All of this suggests that there is significant room for improvement in the department's communications with colleagues, both in promoting the value and place of psychotherapy, and in promoting the exdstence and practice of the service itself.

Interestingly, neither training nor belief in psychotherapy had a significant effect on referral behaviour or knowledge about the department. Perhaps this group was more likely to support and practise psychotherapy within their own 
service, or use the referral process in a different way.

Respondents held stronger views about the structure of the psychotherapy service and its relationship to other psychiatric services. Beliefs that the psychotherapy service should not be a separate service, should not expand, and should not provide all psychotherapy were combined with a view that therapy should be preceded by psychiatric assessment and that responsibility remain with the psychiatrist. This may represent control issues or a simple fear that expansion of psychotherapy would be at the expense of psychiatry.

The potential value of greater communication and an integrated approach was reflected by the numbers of psychiatrists engaged actively in psychotherapy in their own practice, although figures are much lower than those found in another survey (Mowbray \& Timbury, 1966). This may reflect differing cultures or changing patterns of practice over the years, but most said that lack of time was a reason for change in practice in the last three years. Despite the fact that the majority do not think that psychotherapy is a potent treatment modality, considerable numbers are engaged in such treatment and if they are currently doing less of it, it is because they do not have enough time.

Nevertheless, an important point is that local psychiatrists did not know very much about their psychotherapy service and had little contact with it. It is essential to develop services within other districts to help psychotherapy come away from the ivory tower. Broadening the interface between psychiatry and psychotherapy will clarify professional roles and skills, promote better understanding of what is contained in the process of referral and how to use this appropriately. These issues have been identified locally as training needs for GPs and practice counsellors in relation to secondary mental health care. There will of course be a great demand on the service as a training and teaching resource, but the service must retain a role as a specialist treatment facility for those patients who need special expertise or intensive and prolonged treatment.

As psychotherapy training becomes integrated into general psychiatric training in accordance with the College guidelines, more psychiatrists will be informed and trained to practise psychotherapy competently within their own service. From our results it appears that the introduction of the guidelines will, in principle, be welcomed as most feel that psychotherapy should be a part of a psychiatrist's training. Psychotherapy training is currently associated with a psychotherapeutic orfentation to practice, an increase in the amount of time devoted to practice, and a belief in psychotherapy as a potent treatment method. This relationship may not, however, be simple cause and effect. In times when courses and posts on a rotational training scheme are optional it would only be those already interested or curious who would take these up. What will happen when all psychiatrists undergo such training?

Our findings about preferred method of learning psychotherapy are consistent with those of Hook (1973) in that individual supervision is preferred to group supervision. This may reflect the medical training culture. The perceived luxury of the undivided attention of a senior colleague and privacy to struggle with the psychotherapeutic process appears to outweigh the advantages of peer support and cooperative learning. It is our opinion that the group therapeutic factors of cohesion, universality. altruism, interpersonal learning, guidance and modelling (Yalom, 1975) apply to group supervision and have positive ramifications in terms of the trainees' professional development. However, until a culture of open communication about one's reactions to patients prevails, juniors may find group supervision difficult.

Those who have experience in psychotherapy value to a greater degree personal psychotherapy as a training experience. It is part of the curriculum of all formal qualifications. The fact that it is required provides fuel for heated debate among trainers and trainee psychotherapists alike, but the consensus among organisations granting qualifications in psychotherapy is that the personal gains outweigh the disadvantages incurred by its mandatory status. In a survey of psychiatrists who considered themselves to be psychotherapeutically oriented, Hafner et al (1977) found that the majority valued personal insight and believed it had helped them grow and mature as people. However, at present it would be unreasonable to include it in the curriculum of trainee psychiatrists. It might lead to the profession failing to attract otherwise talented doctors.

It seems that on Merseyside, psychiatrists' views of psychotherapy are rather like those towards religion, they don't much believe in it but they don't want to exclude it completely. There is a need for improved communication and training.

\section{References}

Grant, S., Holmes, J. \& Watson, J. (1993) Guidelines for psychotherapy training as part of general professional training. Psychiatric Bulletin, 17, 695-697.

HAFNER, J. LIEBMAN, S. \& CRISP, A. H. (1977) A survey of consultant psychiatrist' attitudes to their work, with particular reference to psychotherapy. British Journal of Psychiatry, 131, 415-419.

Hook. R. H. (1973) Psychotherapy: practice and training, opinions of members of the Australian and New Zealand College of Psychiatrists. Australia and New Zealand Journal of Psychiatry, 7, 87-96.

MOWBRAY, R. M. \& TMmBURY, G. M. (1966) Opinions on psychotherapy: an enquiry. British Joumal of Psychiatry. 112, 351-361. 
YALOM, I. D. (1975) The Theory and Practice of Group Psychotherapy (2nd edn). New York: Basic Books.

* Sue Bradbury, Consultant Psychiatrist, Eastbourne General District Hospital, Eastbourne BN21 2UD; L. S. O'Brien, Consultant Psychiatrist, Huntercombe Manor Hospital, Maidenhead
SL6 OPQ: and Micheal Göpfert, Consultant Psychotherapist, Liverpool Psychotherapy and Consultation Service, Mossley Hill Hospital, Park Lane, Liverpool L18.

-Correspondence 